\section{Benefits of exercise in radiotherapy}

Cancer patients experiencing treatment- and disease-related fatigue are commonly advised to rest and relax, although inactivity may actually be detrimental. To address the lack of randomized, controlled trials of exercise interventions, Windsor et al. have studied the effects of aerobic exercise in patients undergoing radiotherapy for localized prostate carcinoma.

Consecutive patients were randomized to an exercise group ( $n=33)$ or a control group $(n=33)$ before receiving radical external beam radiation therapy. During the 4-week treatment period, patients in the exercise group were advised to carry out home-based, moderate-intensity, continuous walking for 30 minutes at least three times per week. Men in the control group were given 'normal' advice, i.e. to carry out activities as usual and to rest if fatigued. Outcome measures were fatigue (assessed using the Brief Fatigue Inventory $[\mathrm{BFI}]$ ) and physical functioning (assessed by measuring the distance walked in a modified shuttle test).

Fatigue scores increased significantly in the control group after therapy $(P=0.013)$, whereas men in the exercise group showed no significant changes in fatigue over time. The shuttle test distance was reduced by $2.4 \%$ in the control group after therapy, but this was not statistically significant. Physical functioning was significantly improved, however, in the exercise group, whose mean shuttle test distance had increased by $13.2 . \%$ at the end of treatment $(P=0.0003)$.

The authors conclude that home-based, moderate-intensity walking improved physical functioning and that this may be necessary to minimize radiation-induced fatigue.

Original article Windsor PM et al. (2004) A randomized, controlled trial of aerobic exercise for treatment-related fatigue in men receiving radical external beam radiotherapy for localized prostate carcinoma. Cancer 101: 550-557

\section{Prevention of postoperative erections: role for ketoconazole?}

Painful erections are common following penile and urethral reconstructive surgery, but current methods for preventing this problem are ineffective. Evans et al. observed a decrease in postoperative erections when patients were treated with oral ketoconazole, an imidazole derivative that reversibly inhibits testosterone production. They have carried out a retrospective, pilot study to evaluate the efficacy of this approach.

The authors studied the records of patients aged 13-72 years who had undergone penile or urethral surgery during the previous 3 years. Patients who had been prescribed ketoconazole (400 mg three times daily for 10-14 days postoperatively) were labeled the study group $(n=31)$ and those who had not received the drug formed the control group $(n=7)$. The incidence of erections (painful or otherwise) during the immediate postoperative period was compared between the two groups. Overall postoperative pain, surgical outcomes, side effects and patient satisfaction were also compared.

The incidence of postoperative erections was significantly lower in the study group than in the control group ( $23 \%$ vs $71 \%$; $P=0.02)$, as was the incidence of painful erections (16\% vs $71 \% ; P=0.008)$. Surgical outcomes, patient satisfaction and the incidence of overall postoperative pain were comparable between the two groups and side effects in the study group were minimal.

The authors conclude that ketoconazole is effective in preventing painful postoperative erections and they are now investigating this further in a prospective, placebo-controlled, double-blind study.

Original article Evans KC et al. (2004) Use of oral ketoconazole to prevent postoperative erections following penile surgery. Int J Impotence Res 16: 346-349

\section{Magnetic stimulation for urinary incontinence}

Extracorporeal magnetic innervation (ExMl) is a new, noninvasive method for the treatment of stress urinary incontinence, based on the direct stimulation of the pelvic floor muscles and sacral roots. Yokoyama and colleagues have carried out a prospective study to determine whether the technique is also effective in treating urge incontinence.

Patients with urge incontinence $(n=20)$ or stress incontinence $(n=17)$ were treated using the Neocontrol ExMl system (Neotonus Inc., Marietta, GA) for 20 minutes, twice per week for 